\title{
Relações entre idade, peso, ganho médio diário e tempo médio de permanência de novilhos de corte confinados para abate aos 15 ou 27 meses de idade
}

\section{Relationships between age, weight, average weight gain and days on feed of beef steers slaughtered at 15 or 27 months of age}

\author{
Carlos Santos Gottschall $1^{*} ;$ Leonardo Canali Canellas²; \\ Pedro Rocha Marques ${ }^{3}$; Hélio Radke Bittencourt ${ }^{4}$
}

\begin{abstract}
Resumo
Objetivou-se estudar as relações entre idade, peso, ganho médio diário de peso e tempo médio de permanência de novilhos de corte confinados para abate aos 15 ou 27 meses de idade. Foram utilizados 432 novilhos com base racial britânica e cruzas, sendo 267 novilhos superprecoces, confinados aos 12 meses e abatidos em média aos 15 meses (grupo NSP) e 165 novilhos precoces, confinados aos 25 meses e abatidos em média aos 27 meses (grupo NP). Os NSP e os NP iniciaram o experimento com peso médio (PMI) de 296,98 e 352,04 kg, respectivamente ( $\mathrm{p}<0,01)$ e foram terminados em confinamento até que atingissem um grau de terminação adequado. Em virtude disso, ocorreram sete períodos diferentes de abate. Na análise estatística o peso médio inicial (PMI) e o tempo médio de permanência (TMP) foram utilizados como co-variáveis. O grupo NP apresentou maior ( $\mathrm{p}<0,01)$ GMD $(1,351 \times 1,004 \mathrm{~kg} /$ dia) e PMF $(441,88 \times 388,88 \mathrm{~kg})$ e menor $(\mathrm{p}<0,01)$ TMP $(68,87 \times 94,20$ dias, respectivamente $)$. O ganho de peso vivo (GPV) foi semelhante $(\mathrm{p}>0,05)$ entre os grupos $(91,90$ e 89,27 kg para NSP e NP, respectivamente). Animais que apresentaram maiores PMI foram vendidos mais cedo, obtendo menores TMP. Nos dois grupos foi observada uma tendência de queda no GMD com o aumento do TMP. Novilhos precoces obtiveram maior GMD, acarretando em um menor TMP em relação aos superprecoces. Com o aumento do TMP houve redução no GMD de ambos os grupos. Nos dois grupos, animais com maior PMI permaneceram confinados por menos tempo.
\end{abstract}

Palavras-chave: Novilhos precoces, tempo de permanência, ganho de peso, confinamento

\begin{abstract}
The main objective of this research was to evaluate the relationship between age, weight, average weight gain and days on feed of beef steers slaughtered at 15 or 27 months of age. Four hundred and thirty two beef steers were divided in two groups: 267 younger steers (YY), confined at 12 and slaughtered at 15 months old; and 165 young steers (YS), confined at 25 and slaughtered at 27 months of age. Beginning weight (BW) was 296.98 and $352.04 \mathrm{~kg}$ to YY and YS, respectively $(\mathrm{p}<0.01)$. Steers were on feedlot until reach a minimum fat percentage, resulting on seven different values for DF. In the statistical analysis the BW and the DF were calculated as co-variables. YS reach higher AWG (1.351 vs $1.004 \mathrm{~kg} / \mathrm{day})$ and higher SW (441.88 vs $388.88 \mathrm{~kg})(\mathrm{p}<0.01)$ and lower ( $\mathrm{p}<0.01)$ DF (66.87 and 94.20 days, respectively). Steers with higher BW were sold earlier, reaching lower DF. Both groups showed a decrease tendency
\end{abstract}

1 Médico Veterinário, MSc. Professor Adjunto da Faculdade de Medicina Veterinária - ULBRA. E-mail: carlosgott@cpovo.net.

2 Médico Veterinário. Mestrando do Programa de Pós-Graduação em Zootecnia da UFRGS. Bolsista do CNPq.

3 Médico Veterinário. Mestrando em Agronegócios, CEPAN/UFRGS. Bolsista da CAPES.

4 Estatístico, MSc. Faculdade de Matemática, PUCRS.

* Autor para correspondência

Recebido para publicação 16/11/07 Aprovado em 08/05/09 
of AWG as the DF increased. YS reached higher AWG, resulting in lower DF compared with YY. In both groups, while the DF increases, the AWG decreases. Steers with higher BW reached lower DF.

Key words: Young steers, days on feed, weight gain, feedlot

\section{Introdução}

A terminação de bovinos em confinamento é uma das práticas crescentes na pecuária de corte brasileira. No ano de 2006, foram terminados no Brasil aproximadamente 2 milhões e 490 mil bovinos confinados, valor este superior a $8 \%$ em relação ao ano de 2005 (ANUALPEC, 2006). Esse crescimento pode ser caracterizado, em partes, devido à necessidade de melhorias dos baixos índices produtivos apresentados atualmente (PACHECO et al., 2005). Além disso, o confinamento proporciona segurança ao sistema produtivo quando se deseja atingir determinados índices produtivos, pois permite melhor controle da dieta, redução do período de abate e o monitoramento do desempenho dos animais (COSTA et al., 2002).

Segundo Pascoal, Vaz e Roso (1999), o confinamento de bovinos é uma alternativa para aumentar a escala de produção, pois permite a terminação de animais (novilhos e/ou fêmeas de descarte) em uma área reduzida da propriedade, liberando áreas de pastagem para categorias em recria. Além disso, o confinamento pode ser utilizado para a produção de novilhos precoces (abatidos aos 24-30 meses) e superprecoces (abatidos aos 14-16 meses), antecipando receitas e proporcionando giro rápido do capital investido (EUCLIDES FILHO et al., 2003).

O desempenho do animal em confinamento, representado principalmente pela taxa de ganho de peso, é um dos principais fatores que afetam a eficiência do sistema de produção (ALBRIGHT et al., 1994). Di Marco, Barcellos e Costa (2006) afirmam que a taxa de ganho de peso é bifásica, isto é, aumenta com o aumento do peso vivo até que o animal atinge o ponto de engorda, sendo que, após essa fase, o ganho de peso tende a diminuir. Diversos fatores exercem influência sobre essa variável quando se trata de animais em crescimento, entre eles o peso, a idade, o nível nutricional, a genética e o sexo (GOTTSCHALL; CANELLAS; FERREIRA, 2006; LEME; GUEDES, 2005).

O ganho diário de peso apresenta uma correlação negativa com o tempo de permanência dos animais em confinamento, ou seja, o aumento na taxa de ganho de peso gera redução no número de dias de confinamento. Se o ganho de peso diário for semelhante, novilhos mais velhos e com elevado peso corporal necessitarão de um menor tempo para atingir o grau de terminação adequado do que animais mais jovens, pois o ganho de peso necessário para tanto será menor. Ou seja, quanto menor a idade e o peso de entrada no confinamento maior será o tempo de permanência até o abate, o que leva à redução na eficiência do sistema de produção (DI MARCO, 1998; HERSOM et al., 2004). Tendo em vista a hipótese de que novilhos de diferentes idades e pesos tendem a apresentar diferentes desempenhos quando terminados em confinamento, o objetivo do presente trabalho foi o de avaliar as relações entre idade, peso, ganho médio diário e tempo médio de permanência de novilhos de corte confinados para abate aos 15 ou 27 meses de idade.

\section{Materiais e métodos}

O trabalho foi conduzido no município de Carazinho, região do Planalto Médio do Rio Grande do Sul, entre 22/09/2006 e 01/02/2007. Foram utilizados 432 novilhos de corte (base racial Angus e cruzas com Nelore) sendo 267 novilhos confinados aos 12 meses e abatidos em média aos 15 meses de idade, denominados superprecoces (grupo NSP) e 165 novilhos confinados aos 25 meses e abatidos em média aos 27 meses, denominados precoces (grupo NP). Os animais, identificados individualmente, foram pesados ao início do experimento e a cada 30 dias, em média, até a pesagem final por ocasião das vendas. As pesagens 
intermediárias foram utilizadas para avaliação do desempenho dos animais e eventuais ajustes na dieta. Anteriormente às pesagens, realizadas sempre pela manhã, os animais foram submetidos a um jejum de 14 horas. Os animais foram mantidos em confinamento até que atingissem o grau de terminação adequado (escore de condição corporal entre 7 e 8, em uma escala de 1 a 9), expresso por visualização de gordura subcutânea, não tendo peso nem data pré-determinados para o abate. Em virtude disso, ocorreram sete períodos diferentes de abate, numerados em ordem seqüencial (de 1 a 7).

Durante os 12 primeiros dias de confinamento, os animais foram submetidos a uma adaptação à dieta, ajustada conforme National Research Council NRC (1996), para GMD de 1,1 kg/dia. O volumoso utilizado era composto de quantidades variáveis de silagens de milho, girassol, laranja e aveia, e o concentrado continha farelos de soja e girassol, resíduos de soja e trigo, grãos de milho e sorgo, calcário calcítico e monensina sódica (1,5 gramas por animal/dia), na relação volumoso:concentrado de 54:46 (Tabelas 1 e 2). Por questão operacional, foi utilizada uma dieta única para ambos os grupos, sendo ajustado o fornecimento da mesma em quantidades diferentes para cada grupo. A dieta era fornecida na base de 3,0\% (MS) do peso vivo e continha valores médios de $70 \%$ de nutrientes digestíveis totais (NDT) e 12,5\% de proteína bruta $(\mathrm{PB})$, e era fornecida duas vezes ao dia, às 8 e às 16 horas, em cochos com espaço linear de $50 \mathrm{~cm}$ por animal. O manejo sanitário seguiu o calendário normal da propriedade, visando o controle de endoparasitas e ectoparasitas.

Tabela 1. Composição do volumoso in natura utilizado para NSP (novilhos superprecoces) e NP (novilhos precoces) nos diferentes períodos de confinamento (em kg).

\begin{tabular}{lcccccc}
\hline \multirow{2}{*}{ Volumoso (in natura) } & \multicolumn{2}{c}{ Período I } & \multicolumn{2}{c}{ Período II } & \multicolumn{2}{c}{ Período III } \\
& \multicolumn{2}{c}{$(\mathbf{2 2 / 9}$ a 20/11) } & \multicolumn{2}{c}{$(\mathbf{2 1 / 1 1}$ a 16/12) } & \multicolumn{2}{c}{$(\mathbf{1 7 / 1 2}$ a 01/02) } \\
& NSP & NP & NSP & NP & NSP & NP \\
\hline Silagem de aveia $(\mathrm{kg})$ & 6,3 & 6,7 & 6,3 & 6,7 & 6,3 & 6,7 \\
Silagem de laranja $(\mathrm{kg})$ & 4,9 & 5,2 & 4,9 & 5,2 & 5,8 & 6,2 \\
Silagem de girassol $(\mathrm{kg})$ & 2,9 & 3,1 & 2,9 & 3,1 & 2,9 & 3,1 \\
Silagem de milho $(\mathrm{kg})$ & 2,9 & 3,1 & 2,9 & 3,1 & 2,9 & 3,1 \\
\hline Total $(\mathrm{kg})$ & 17,0 & 18,4 & 17,0 & 18,4 & 17,9 & 19,1 \\
\hline
\end{tabular}

Tabela 2. Composição do concentrado in natura utilizado para NSP (novilhos superprecoces) e NP (novilhos precoces) nos diferentes períodos de confinamento (em $\mathrm{kg}$ ).

\begin{tabular}{lcccccc}
\hline & \multicolumn{2}{c}{ Período I } & \multicolumn{2}{c}{ Período II } & \multicolumn{2}{c}{ Período III } \\
Concentrado (in natura) & \multicolumn{2}{c}{$\mathbf{( 2 2 / 9}$ a 20/11) } & \multicolumn{2}{c}{$\mathbf{( 2 1 / 1 1}$ a 16/12) } & \multicolumn{2}{c}{$(\mathbf{1 7 / 1 2}$ a 01/02) } \\
& NSP & NP & NSP & NP & NSP & NP \\
\hline Farelo de soja (kg) & 0,33 & 0,35 & 0,46 & 0,48 & 0,58 & 0,62 \\
Resíduo de soja (kg) & 0,83 & 0,89 & - & - & - & - \\
Farelo de girassol (kg) & 0,38 & 0,40 & 0,33 & 0,35 & - & - \\
Milho grão (kg) & 0,70 & 0,74 & 0,39 & 0,41 & 0,89 & 0,95 \\
Sorgo grão (kg) & 1,15 & 1,22 & 0,88 & 0,94 & - & - \\
Resíduo de trigo (kg) & 1,05 & 1,11 & - & - & - & - \\
Resíduo de cevada (kg) & - & - & 2,11 & 2,25 & 2,55 & 2,89 \\
Farelo de arroz & - & - & 0,37 & 0,39 & 0,74 & 0,78 \\
Calcário calcítico $(\mathrm{kg})$ & 0,15 & 0,16 & 0,13 & 0,14 & 0,13 & 0,14 \\
\hline Total $(\mathrm{kg})$ & 4,59 & 4,87 & 4,67 & 4,96 & 4,89 & 5,38 \\
\hline
\end{tabular}


A análise estatística consistiu da utilização do módulo de Modelos Lineares Generalizados do pacote SPSS versão 10.0 para comparação do ganho médio diário de peso (GMD) entre NSP e NP, utilizando o peso médio inicial (PMI) e o tempo médio de permanência (TMP) como covariáveis. Também foram utilizados os testes $t$ de Student e Tukey (níveis de significância de 1 e 5\%) para comparação dentro das categorias entre cada venda. Segundo o modelo linear para comparação do GMD entre NSP e NP, verificou-se que o tempo de permanência e a categoria exercem influência significativa sobre o GMD. Estimativas para o GMD podem ser geradas a partir do seguinte modelo:

$$
\begin{gathered}
\mathrm{GMD}=1,870+0,000238 \times \mathrm{PMI}-0,008756 \mathrm{x} \\
\text { TMP }-0,11232 \times \text { grupo. } \\
\text { Grupo: } \mathrm{NSP}=1 \mathrm{e} \mathrm{NP}=0
\end{gathered}
$$

\section{Resultados e discussão}

Na Tabela 3 podem ser visualizados os valores obtidos para peso médio inicial (PMI) em kg, peso médio final (PMF) em $\mathrm{kg}$, tempo médio de permanência (TMP) em dias, ganho médio diário de peso (GMD) em kg/dia e ganho de peso vivo (GPV) em kg.

Tabela 3. Peso médio inicial (PMI) em kg, peso médio final (PMF), tempo médio de permanência (TMP), ganho médio diário de peso (GMD) e ganho de peso vivo (GPV), de novilhos superprecoces (NSP) e precoces (NP) submetidos ao confinamento.

\begin{tabular}{lcc}
\hline Variável & NSP $(\mathbf{n}=\mathbf{2 6 7})$ & $\mathbf{N P}(\mathbf{n}=\mathbf{1 6 5})$ \\
\hline PMI $(\mathrm{kg})$ & $296,98 \mathrm{a}$ & $352,04 \mathrm{~b}$ \\
PMF (kg) & $388,88 \mathrm{a}$ & $441,88 \mathrm{~b}$ \\
TMP (dias) & $94,20 \mathrm{a}$ & $68,87 \mathrm{~b}$ \\
GMD (kg/dia) & $1,004 \mathrm{a}$ & $1,351 \mathrm{~b}$ \\
GPV (kg) & 91,90 & 89,27 \\
\hline
\end{tabular}

a,b na linha diferem entre si $(\mathrm{p}<0,01)$.

Os novilhos precoces apresentaram maior $(\mathrm{p}<0,01)$ PMI do que os superprecoces, e o GPV se apresentou semelhante $(p>0,05)$ para os dois grupos, o que explica o fato do PMF ter sido superior $(p<0,01)$ para os NP em relação aos NSP. Resultados semelhantes foram encontrados por Gottschall et al. (2007), nos quais os novilhos superprecoces com maior peso inicial obtiveram maior peso ao abate. Resultados esses semelhantes a diversos autores, pelos quais afirmam que o peso de terminação diminui com o aumento do plano nutricional (COLEMAN; EVANS; GENTHER, 1993; DI MARCO; BARCELLOS; COSTA, 2006). Esse maior aporte de nutrientes, por sua vez, deve ocorrer sempre que o objetivo for o abate de novilhos com até 14 meses de idade, visto que, em um curto período, a necessidade de ganho de peso dessa categoria animal é elevada, aumentando também sua exigência nutricional. Deste modo, bezerros submetidos a dietas que proporcionem altas taxas de ganho de peso tendem a atingir o ponto de terminação precocemente e com menores pesos em relação a novilhos mais velhos. Em contrapartida, Gottschall (2005) ressalta que para a produção de novilhos precoces existe uma grande flexibilidade quanto ao momento e à intensidade do ganho de peso. Novilhos abatidos entre 24-30 meses exigem GMD moderado $(0,500 \mathrm{~kg} /$ dia $)$ devendo este ser mais intenso na fase final de terminação, que muitas vezes coincide com o confinamento. Com isso, novilhos precoces tendem a apresentar pesos mais elevados e maior estrutura corporal por ocasião da entrada no confinamento em relação aos superprecoces. Esse fato também contribuiu para o maior PMF 
verificado nos NP, visto que esses apresentaram GPV semelhante ao dos novilhos superprecoces (NSP), como relatado anteriormente.

Vaz e Restle (2003) verificaram que novilhos precoces e superprecoces apresentam GMD semelhantes quando submetidos à terminação intensiva recebendo dietas com qualidade semelhante. Entretanto, no presente trabalho o GMD foi maior $(\mathrm{p}<0,01)$ para os NP, o que possivelmente ocorreu devido à maior estrutura corporal desses animais em relação aos superprecoces por ocasião do início do confinamento, resultando em um maior ganho compensatório para esses animais. Conforme estudos de Hersom et al. (2004) animais que sofreram algum tipo de restrição alimentar prévia tendem a fazer um ganho ou crescimento compensatório quando passam a receber dietas de melhor qualidade. Segundo Medeiros e Lana (2000), o aumento no consumo voluntário de alimentos, a redução da mantença, a melhor eficiência metabólica e mudanças na composição do ganho são fatores que explicam a compensação no ganho de peso durante esse período. Bail, Brondani e Restle (2000), avaliaram o desempenho em confinamento de novilhos cruzas Charolês e Nelore, com média de 20 meses de idade, mantidos na fase de recria em pastagem nativa ou cultivada, verificaram que o ganho de peso médio diário dos novilhos provenientes da pastagem nativa foi $20 \%$ superior ao daqueles da pastagem cultivada $(1,30 \times 1,08 \mathrm{~kg}$, respectivamente). Choat et al. (2003), comparando o desempenho em confinamento de novilhos manejados previamente em pastagens de baixa ou alta qualidade, relatam maiores taxas de ganho de peso para os animais submetidos à pastagem de pior qualidade, atribuindo o resultado a manifestação do ganho compensatório. Do mesmo modo, Pacheco et al. (2005), trabalhando com novilhos precoces e superprecoces, também encontraram maior GMD para os novilhos precoces $(1,94 \times 1,53 \mathrm{~kg} /$ dia, respectivamente), atribuindo esse resultado ao maior ganho compensatório obtido pelos novilhos precoces. Os valores obtidos para GMD no trabalho de Pacheco et al. (2005) são superiores aos do presente experimento para as duas categorias.

O tempo médio de permanência (TMP) dos novilhos em confinamento foi de 94,20 e 68,87 dias para NSP e NP, respectivamente $(p<0,01)$. Esses períodos de confinamento estão de acordo com os relatados por Lanna eAlmeida (2005), que afirmaram que no Brasil o tempo médio de permanência de bovinos quando confinados fica em torno de 70 a 80 dias. Novilhos precoces permaneceram confinados por um menor $(p<0,01)$ período de tempo em relação aos superprecoces (tabela 3). Apesar de ambos os grupos terem apresentado GPV semelhantes, o GMD foi superior para os NP, o que contribuiu para que esses novilhos atingissem mais rapidamente seu peso de terminação (PMF), acarretando na antecipação do abate desses animais em cerca de 25 dias quando comparados aos NSP.

$\mathrm{Na}$ Tabela 4 podem ser visualizados os GMD de novilhos precoces e superprecoces conforme a seqüência de venda (de 1 a 7). Os resultados demonstram que além do GMD, a intensidade de ganho de peso, provavelmente influenciada pelo ganho compensatório, se manifestou de maneira mais acentuada nos NP. Na primeira venda, 52 dias após a entrada saíram 29\% dos animais do grupo NP e justamente os animais que apresentaram o maior GMD. Na venda 2, o grupo de animais NP apresentou maior $(p<0,05)$ GMD em relação aos NSP. Nesse sentido, Lanna e Almeida (2005) afirmam que no caso de períodos curtos de confinamento (70 a 80 dias) os animais confinados permanecem em crescimento compensatório da chegada ao cocho até o abate, o que está de acordo com o comportamento apresentado pelos animais do presente trabalho, principalmente pelos novilhos precoces. Como relatado anteriormente, a produção de novilhos para abate aos 24-27 meses não exige um manejo nutricional tão intensivo quanto aquele empregado aos superprecoces. Desse modo, esses novilhos acabam sendo submetidos a períodos de restrição alimentar de curta a média duração, geralmente seguidos pelo período final de 
terminação, onde o aporte nutricional é elevado. Em contrapartida, novilhos abatidos em torno de 14-15 meses (superprecoces) necessitam de um ganho de peso contínuo e próximo a 1,0 kg/ dia, ou seja, a produção desse tipo de animal não tolera restrição alimentar que ocasione perda de peso (GOTTSCHALL, 2005). Desse modo, o crescimento compensatório tende a se manifestar mais intensamente em animais mais velhos, ou seja, com estrutura corporal mais desenvolvida, mas que em algum momento sofreram limitação nutricional, mesmo que de baixa intensidade.

Tabela 4. Ganho médio diário de peso (GMD) de novilhos precoces (NSP) e superprecoces (NP) entre as vendas dentro de cada grupo e entre os grupos (NSP e NP).

\begin{tabular}{ccccc}
\hline \multirow{2}{*}{ Venda } & \multicolumn{2}{c}{ NSP } & n & NP \\
\cline { 2 - 5 } & $\mathbf{n}$ & GMD (kg) & 48 & GMD (kg) \\
\hline 1 & - & - & 51 & $1,597 \mathrm{a}$ \\
2 & 13 & $1,166 \mathrm{abA}$ & 26 & $1,425 \mathrm{abB}$ \\
3 & 41 & $1,200 \mathrm{a}$ & 5 & $1,235 \mathrm{~b} \mathrm{c}$ \\
4 & 59 & $1,078 \mathrm{ab}$ & 27 & $1,097 \mathrm{bc}$ \\
5 & 74 & $1,021 \mathrm{~b}$ & 3 & $1,080 \mathrm{bcB}$ \\
6 & 53 & $0,858 \mathrm{cA}$ & 5 & $0,812 \mathrm{c}$ \\
7 & 27 & $0,707 \mathrm{~d}$ & 165 & $1,351 \mathrm{~B}$ \\
\hline
\end{tabular}

a,b na coluna, diferem entre si $(\mathrm{p}<0,05)$.

A,B na linha, diferem entre si $(\mathrm{p}<0,05)$.

Observa-se que, tanto NSP quanto NP apresentaram maiores taxas de ganho de peso nas primeiras vendas, havendo um decréscimo do GMD à medida que aumentou o TMP, conforme mostram a Tabela 4 e as figuras 1 e 2. Observa-se que a inclinação das retas é muito semelhante, indicando que, em média, a cada dia a mais de permanência em confinamento, o GMD tende a diminuir $0,009 \mathrm{~kg} /$ dia. A superioridade do GMD, quando comparados os valores obtidos para essa variável pelo primeiro e pelo último lote de venda, dentro de cada grupo, foi de 39 e $49 \%$ para NSP e NP, respectivamente.

Diversos autores afirmam que com o aumento do tempo de alimentação e da condição corporal do animal, existe uma tendência de queda do ganho de peso (DI MARCO, 1998; GOTTSCHALL, 2005). Isso ocorre devido ao maior acúmulo de tecido adiposo em relação ao tecido muscular, o que gera um maior gasto energético por unidade de ganho de peso. Trabalhando com novilhos superprecoces da raça Angus abatidos de forma escalonada e com diferentes pesos, Costa et al. (2002) verificaram que na medida em que aumentou o tempo de permanência no confinamento houve uma tendência de queda na taxa de ganho de peso, atingindo GMD de 1,32, 1,27, 1,23 e 1,15 kg/dia quando mantidos 114, 144, 168 e 209 dias em confinamento, respectivamente. Costa et al. (2002) atribuíram esses resultados à maior energia gasta pelos animais para manutenção e ganho de peso e pela maior demanda de energia em função da composição do ganho. Nesse sentido, Leme e Guedes (2005) explicam que a curva típica de crescimento de um bovino inicia-se com um ganho de peso acelerado até atingir o ponto onde a taxa de crescimento é máxima. A partir daí, passa a haver uma diminuição no crescimento, com aumento crescente da taxa de deposição de gordura. Essas afirmações corroboram com a tendência de queda na taxa de ganho de peso apresentada com o aumento do tempo de permanência, verificada no presente trabalho. É importante ressaltar que no trabalho de Costa et al. (2002), os animais foram abatidos com datas pré-determinadas, independentemente 
de peso ou acabamento, enquanto que no presente trabalho o abate foi realizado conforme os animais atingiam o grau de acabamento adequado, exigido pelo frigorífico. Mesmo assim, o comportamento do GMD foi semelhante nos dois experimentos relatados, evidenciando uma queda na taxa de ganho de peso com o aumento do tempo de confinamento, como discutido anteriormente.

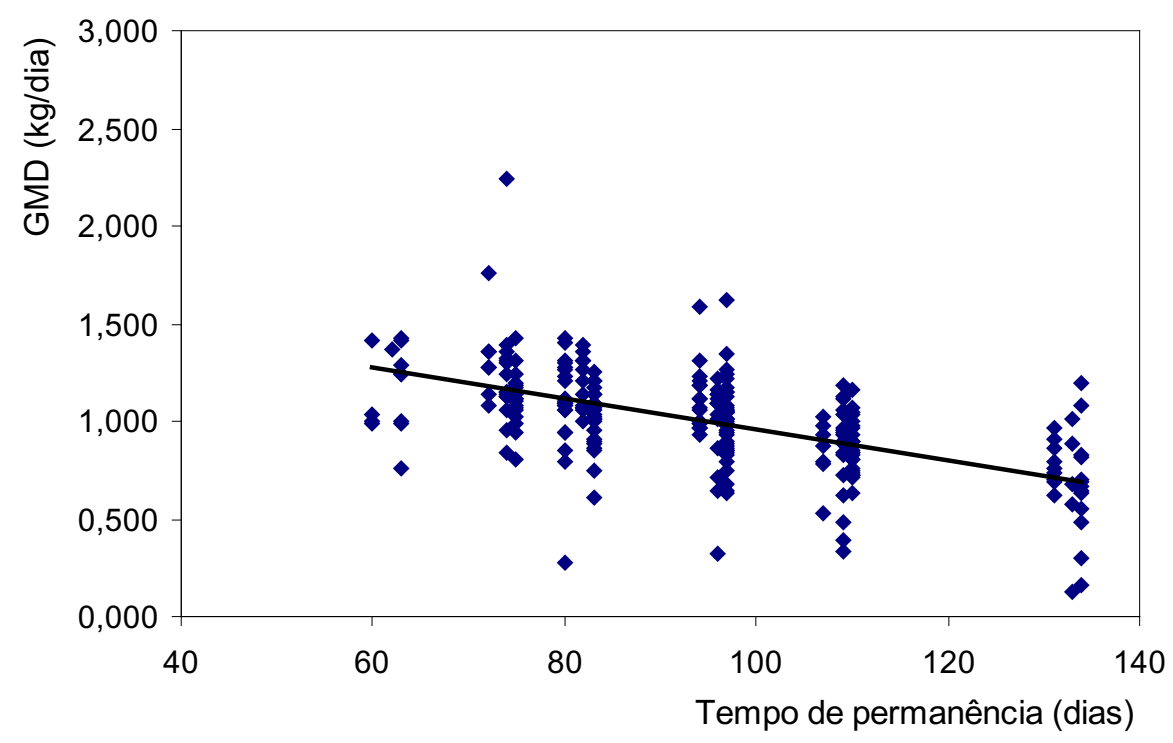

Figura 1. Gráfico de dispersão entre tempo médio de permanência (TMP) e ganho médio diário de peso (GMD) de novilhos superprecoces (NSP) submetidos ao confinamento.

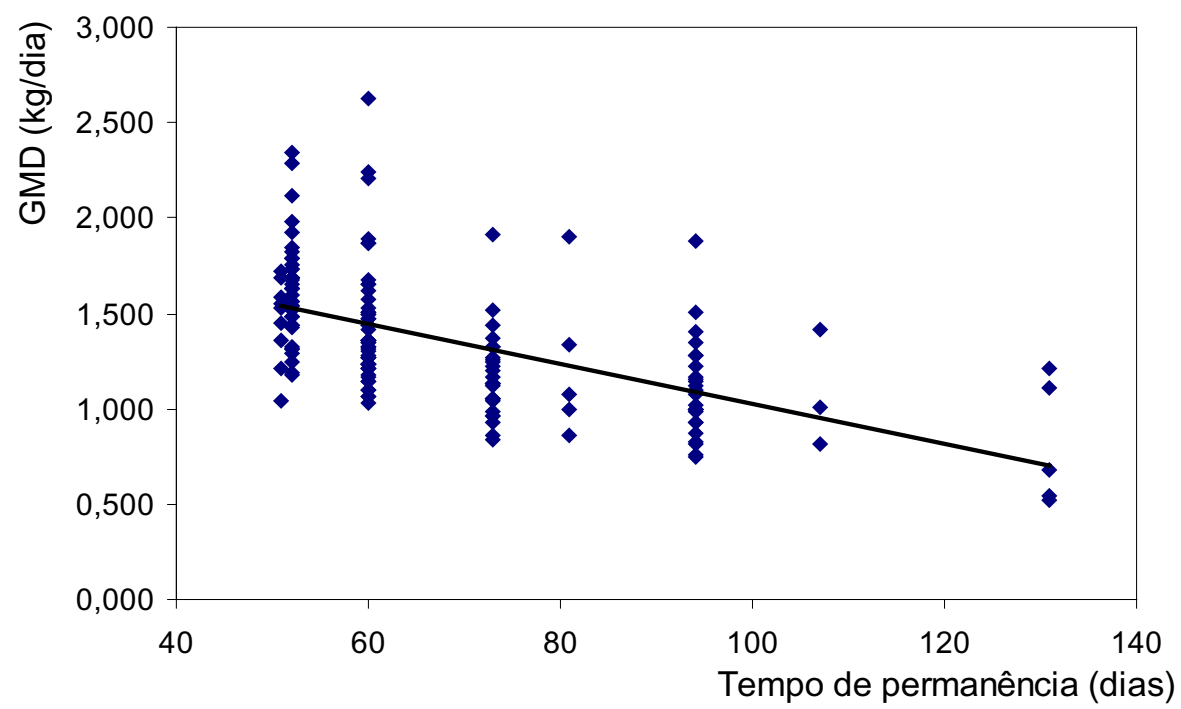

Figura 2. Gráfico de dispersão entre tempo médio de permanência (TMP) e ganho médio diário de peso (GMD) de novilhos precoces (NP) submetidos ao confinamento. 
As Tabelas 5 e 6 mostram o peso médio inicial (PMI), peso médio final (PMF), tempo médio de permanência (TMP), ganho médio diário de peso (GMD) e ganho de peso vivo (GPV), comparando os valores obtidos por cada um dos lotes de venda dentro de cada grupo (NSP e NP). Observa-se que o PMI dos novilhos vendidos nos primeiros lotes foi maior do que o PMI dos animais vendidos nos últimos lotes, tanto dentro do grupo NSP quanto no grupo NP. Dentro do grupo NSP, os lotes 2 e 3 apresentaram maiores PMI em relação aos lotes 5,6 e $7(p<0,01)$ enquanto o lote 4 não diferiu dos demais ( $>>0,05)$. No caso dos NP, os lotes $1,2,3,4$, 5 e 6 não diferiram entre si $(p>0,05)$ e apresentaram maior $(p<0,01)$ PMI em relação ao lote 7 . Esses resultados indicam uma relação entre peso ao início do confinamento e tempo de permanência no confinamento. No presente trabalho quanto maior foi o peso ao início do confinamento menor foi o tempo de permanência no cocho. No grupo NSP, o PMF do lote 3 foi superior $(\mathrm{p}<0,01)$ aos lotes 5,6 e 7, enquanto 2 e 4 não diferiram dos demais $(\mathrm{p}>0,05)$. O grupo NP não apresentou diferença estatística entre os diferentes lotes de venda em relação ao PMF ( $>>0,05)$, o que indica uma maior uniformidade nos pesos de abate dos animais dessa categoria quando comparados aos NSP. Cabe ressaltar, no entanto, que a escolha dos animais a serem abatidos esteve atrelada exclusivamente ao grau de acabamento, estando esse critério sujeito a variações individuais dentro da mesma idade, principalmente no que se refere à relação entre peso e grau de deposição de gordura e tempo necessário para atingir o acabamento desejado.

Tabela 5. Peso médio inicial (PMI) em kg, peso médio final (PMF) em kg, tempo médio de permanência (TMP) em dias, ganho médio diário de peso (GMD) em kg/dia e ganho de peso vivo (GPV) em kg por lote de venda (2 a 7) de novilhos superprecoces (NSP) submetidos ao confinamento.

\begin{tabular}{ccccccc}
\hline Venda & N & PMI $(\mathbf{k g})$ & PMF $(\mathbf{k g})$ & TMP $($ dias) & GMD (kg/dia) & GPV (kg) \\
\hline 2 & 13 & $319,15 \mathrm{a}$ & $391,46 \mathrm{ab}$ & 62,0 & $1,166 \mathrm{ab}$ & $72,31 \mathrm{~b}$ \\
3 & 41 & $317,71 \mathrm{a}$ & $406,61 \mathrm{a}$ & 74,0 & $1,200^{\mathrm{a}}$ & $88,90 \mathrm{a}$ \\
4 & 59 & $302,69 \mathrm{ab}$ & $390,66 \mathrm{ab}$ & 82,0 & $1,078 \mathrm{ab}$ & $87,98 \mathrm{a}$ \\
5 & 74 & $286,30 \mathrm{~b}$ & $384,41 \mathrm{~b}$ & 96,0 & $1,021 \mathrm{~b}$ & $98,11 \mathrm{a}$ \\
6 & 53 & $288,00 \mathrm{~b}$ & $381,72 \mathrm{~b}$ & 109,0 & $0,858 \mathrm{c}$ & $93,72 \mathrm{a}$ \\
7 & 27 & $289,30 \mathrm{~b}$ & $383,11 \mathrm{~b}$ & 133,0 & $0,707 \mathrm{c}$ & $93,82 \mathrm{a}$ \\
\hline Total/Média & 267 & 296,98 & 388,88 & 94,20 & 1,004 & 91,90 \\
\hline
\end{tabular}

a,b na coluna diferem entre si $(\mathrm{p}<0,01)$.

Tabela 6. Peso médio inicial (PMI) em kg, peso médio final (PMF) em kg, tempo médio de permanência (TMP) em dias, ganho médio diário de peso (GMD) em kg/dia e ganho de peso vivo (GPV) em kg por lote de venda (1 a 7) de novilhos precoces (NP) submetidos ao confinamento.

\begin{tabular}{ccccccc}
\hline Venda & N & PMI (kg) & PMF (kg) & TMP (dias) & GMD (kg/dia) & GPV (kg) \\
\hline 1 & 48 & $362,81 \mathrm{ab}$ & $445,604 \mathrm{a}$ & 52,0 & $1,597^{\mathrm{a}}$ & $82,79 \mathrm{c}$ \\
2 & 51 & $355,94 \mathrm{ab}$ & $442,843 \mathrm{a}$ & 60,0 & $1,425 \mathrm{ab}$ & $85,50 \mathrm{bc}$ \\
3 & 26 & $343,15 \mathrm{ab}$ & $428,538 \mathrm{a}$ & 73,0 & $1,174 \mathrm{bc}$ & $85,72 \mathrm{bc}$ \\
4 & 5 & $341,40 \mathrm{ab}$ & $441,400 \mathrm{a}$ & 81,0 & $1,235 \mathrm{ab}$ & $100,00 \mathrm{abc}$ \\
5 & 27 & $342,89 \mathrm{ab}$ & $447,074 \mathrm{a}$ & 94,0 & $1,097 \mathrm{bc}$ & $103,09 \mathrm{abc}$ \\
6 & 3 & $330,00 \mathrm{ab}$ & $446,667 \mathrm{a}$ & 107,0 & $1,080 \mathrm{bc}$ & $115,59 \mathrm{a}$ \\
7 & 5 & $328,40 \mathrm{~b}$ & $435,400 \mathrm{a}$ & 131,0 & $0,812 \mathrm{c}$ & $106,40 \mathrm{ab}$ \\
\hline Total/Média & 165 & 352,04 & 441,89 & 68,87 & 1,351 & 89,25 \\
\hline
\end{tabular}

a,b, c na coluna diferem entre si $(\mathrm{p}<0,01)$. 


\section{Conclusões}

Novilhos precoces obtiveram maior ganho médio diário de peso, acarretando em um menor tempo de permanência em confinamento em relação aos superprecoces. Nos dois grupos, houve uma tendência de queda no ganho médio diário de peso com o aumento do tempo de permanência, manifestando-se inicialmente de forma mais intensa dentro do grupo de novilhos precoces. Fato este que ocorreu possivelmente, a um maior ganho compensatório realizado por esses animais em relação aos superprecoces. Em ambos os grupos, os animais que apresentaram maior peso médio inicial atingiram o grau de terminação mais cedo, permanecendo por menos tempo confinados.

\section{Referências}

ALBRIGHT, M. L.; LANGEMEIER, M. R.; MINTERT, J. R.; SCHROEDER, T. C. Factors affecting cattle feeding profitability and cost of gain. Manhattan, 1994. (Beef Cattle Handbook, BCH-8050).

ANUALPEC 2006: Anuário da Pecuária Brasileira. São Paulo: Instituto FNP, 2006.

BAIL, C. A. T.; BRONDANI, I. L.; RESTLE, J. Níveis de concentrado na fase de terminação em confinamento para novilhos previamente mantidos em pastagem nativa ou cultivada. Ciência Rural, Santa Maria, v. 30, n.1, p. 151-157, 2000.

CHOAT, W. T.; KREHBIEL, C. R.; DUFF, G. C.; KIRKSEY, E. E.; LAURIAULT, L. M.; RIVERA, J. D.; CAPITAN, B. M.; WALKER, D. A.; DONART, G. B.; GOAD, C. L. Influence of grazing dormant native range or winter wheat pasture on subsequent finishing cattle performance, carcass characteristics, and ruminal metabolism. Journal of Animal Science, Champaign, v. 81, n. 12, p. 3191-3201, 2003.

COLEMAN, S. W.; EVANS, B. C.; GUENTHER, J. J. Body and carcass composition of Angus and Charolais steers as affected by age and nutrition. Journal of Animal Science, Champaign, v. 71, n. 1, p. 86-95, 1993.

COSTA, E. C.; RESTLE, J.; PASCOAL, L. L.; VAZ, F. N.; ALVES FILHO, D. C.; ARBOITTE, M. Z. Desempenho de novilhos Red Angus superprecoces, confinados e abatidos com diferentes pesos. Revista Brasileira de Zootecnia, Viçosa, v. 31, n. 1, p. 129-138, 2002.
DI MARCO, O. N. Crecimiento de vacunos para carne. Balcarce: Oscar N. Di Marco, 1998.

DI MARCO, O. N.; BARCELLOS, J. O. J.; COSTA, E. C. Crescimento de bovinos de corte. Porto Alegre: Universidade Federal do Rio Grande do Sul, 2006.

EUCLIDES FILHO, K.; FIGUEIREDO, G. R.; EUCLIDES, V. P. B.; SILVA, L.O.C.; ROCCO, V.; BARBOSA, R. A.; JUNQUEIRA, C. E. Desempenho de diferentes grupos genéticos de bovinos de corte em confinamento. Revista Brasileira de Zootecnia, Viçosa, v. 32, n. 5, p. 1114-1122, 2003.

GOTTSCHALL, C. S. Produção de novilhos precoces: nutrição, manejo e custos de produção. 2. ed. Guaíba: Agrolivros, 2005.

GOTTSCHALL, C. S.; CANELLAS, L. C.; FERREIRA, E. T. Confinamento de bovinos de corte: alternativas para o aumento da eficiência econômica. In: GOTTSCHALL, C. S.; SILVA, J. L. Anais do XI Ciclo de Palestras em Produção e Manejo de Bovinos. Canoas: Ed. da ULBRA, 2006, p. 57-66.

GOTTSCHALL, C. S.; CANELLAS, L. C.; FERREIRA, E. T.; BITTENCOURT, H. R. Desempenho de novilhos Angus, Devon e cruzas Angus x Devon x Nelore em confinamento. Semina: Ciências Agrárias, Londrina, v. 28, n.1, p. 135-142, 2007.

HERSOM, M. J.; HORN, G. W.; KREHBIEL C. R.; PHILLIPS, W. A. Effect of live weight gain of steers during winter grazing: I. Feedlot performance, carcass characteristics, and body composition of beef steers. Journal of Animal Science, Champaign, v. 82, n. 1, p. 262-272, 2004.

LANNA, D. P. D.; ALMEIDA, R. A terminação de bovinos em confinamento. Visão Agrícola, Piracicaba, $\mathrm{n}$. 3, p. 55-58, 2005.

LEME, P. R.; GUEDES, C. Crescimento eficiente do animal pode aumentar rentabilidade. Visão Agrícola, Piracicaba, n. 3, p. 37-39, 2005

MEDEIROS, S. R.; LANNA, D. P. D. Crescimento compensatório em bovinos. 2000. Palestra apresentada no Simpósio Nutron de Nutrição de Ruminantes (GoiâniaGO).

NATIONAL RESEARCH COUNCIL - NRC. Nutrient requeriments of beff cattle. 7. ed. Washington: National Academy Press, 1996.

PACHECO, P. S.; RESTLE, J.; SILVA, J. H. S.; BRONDANI, I. L.; PASCOAL, L. L.; ARBOITTE, M. Z.; FREITAS, A. K. Desempenho de novilhos jovens e superjovens de diferentes grupos genéticos terminados 
em confinamento. Revista Brasileira de Zootecnia, Viçosa, v. 34, n. 3, p. 963-975, 2005.

PASCOAL, L. L.; VAZ, R. Z.; ROSO, C. Confinamento versus pastagem na terminação de bovinos. In: RESTLE, J. (Ed.). Confinamento, pastagens e suplementação para produção de bovinos de corte. Santa Maria: UFSM, 1999. p. 178-190.
VAZ, F. N.; RESTLE, J. Ganho de peso antes e após os sete meses no desenvolvimento e nas características de carcaça e carne de novilhos Charolês abatidos aos dois anos. Revista Brasileira de Zootecnia, Viçosa, v. 32, n. 3, p. 699-708, 2003. 